Pädiatrie up2date

\title{
$2 \cdot 2018$
}

\section{Entwicklung 1}

\section{Elektronische Medien und frühe Kindheit}

VNR: 2760512018154654321

DOI: $10.1055 / \mathrm{s}-0043-115286$

Pädiatrie up2date 2018; 13 (2): 119-134

ISSN 1611-6445

(c) 2018 Georg Thieme Verlag KG 


\section{Unter dieser Rubrik sind bereits erschienen:}

Das späte Frühgeborene - unterschätzte Morbidität R. Klein, A. Kribs $1 / 2018$

Primärer vesikoureteraler Reflux R. Beetz 3/2016

Psychosomatische Störungen im Kindes- und Jugendalter

S. Towfigh, L. Kuntze, M. von Aster 2/2016

Perinatale Programmierung E. Nüsken, F. Lechner,

K.-D. Nüsken 1/2016

Vorsorgeuntersuchungen bei Jugendlichen - Teil 2

T. Baumann 3/2015

Vorsorgeuntersuchungen bei Jugendlichen - Teil 1

T. Baumann 2/2015

Chronischer Mittelohrerguss $M$. Jungehülsing, C. Erle-Bischoff $4 / 2013$

Wie Kinder die Welt abbilden - und was man daraus

folgern kann O. Jenni 3/2013
Wachstumsschmerzen H.-I. Huppertz 2/2013

„Floppy Baby“ A. Klein, E. Boltshauser 4/2011

Asperger-Syndrom R. Gundelfinger 3/2011

Das Entwicklungsprofil O. Jenni, C. Benz, B. Latal 2/2011

Die Entdeckung der Sprache B. Zollinger 3/2010

Entwicklungspädiatrische Säuglingsuntersuchung

T. Baumann 1/2009

Motorik im Schulalter O. Jenni, J. Caflisch, B. Latal 4/2008

Gedeihstörungen M. Rhein, M. Knuf 2/2008

Schlafstörungen O. Jenni, C. Benz 4/2007

Vesikoureteraler Reflux H. Bachmann 3/2007

\section{ALLES ONLINE LESEN}

Mit der eRef lesen Sie Ihre Zeitschrift: online wie offline, am PC und mobil, alle bereits erschienenen Artikel. Für Abonnenten kostenlos! https://eref.thieme.de/paed-u2d

\section{JETZT FREISCHALTEN}

Sie haben Ihre Zeitschrift noch nicht freigeschaltet? Ein Klick genügt: www.thieme.de/eref-registrierung 


\title{
Elektronische Medien und frühe Kindheit
}

Volker Mall, Frank W. Paulus

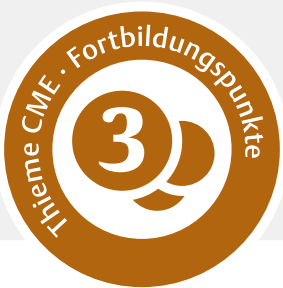

\begin{abstract}
Welche Bedeutung haben die neuen Medien und die zunehmende Medialisierung der kindlichen Lebenswelten für die Kindesentwicklung und Kindergesundheit unter kognitiven, emotionalen, sozialen und physischen Aspekten? Inwiefern können moderne elektronische Medien entwicklungsspezifischen Anforderungen gerecht werden? Und überhaupt - kann ihre Nutzung im Kindesalter empfohlen werden? Der Beitrag bewertet hierzu interessante Studien.
\end{abstract}

\section{Einführung}

Während sich in den 1950er- und 1960er-Jahren das Freizeitverhalten durch die massenhafte Verbreitung des Fernsehens radikal veränderte, sind die Auswirkungen des Internets, der mobilen Nutzergeräte und der Softwareentwicklungen (wie Computerspiele, Kommunikationsprogramme und andere Apps) auf unsere Gesellschaft heute als viel umfassender und tiefgreifender anzusehen. Die Nutzung der neuen Medien ist nicht nur in der Freizeitgestaltung fest etabliert, sie ist in der Arbeitswelt nahezu aus keinem Bereich mehr wegzudenken und macht ein zentrales Element der Kommunikation und alltäglichen Beschäftigungen aus (z.B. Spielen, Warenbestellungen, Online-Banking, Partnersuche, Informationsquellen suchen und Rezipieren der Massenmedien auch unterwegs und auf Reisen). Auf den Markt kommen ständig neue Anwendungen und Endgeräte. Die Technik wird mobiler, vielseitiger, immer mehr vernetzt und gewissermaßen „kinderleicht“ bedienbar ( $\mathbf{A b b} \mathbf{1}$ ).

\section{Multimediakonsum und Entwicklung}

Inzwischen gibt es große Kohortenstudien (z. B. [1, 2]), womit dezidierte evidenzbasierte Aussagen zur Auswirkung von Medienkonsum auf die Kindesentwicklung und -gesundheit getroffen werden können. Dennoch ist es an dieser Stelle notwendig, die wichtigsten Elemente der Kindesentwicklung und grundsätzliche Einflüsse zunächst isoliert zu betrachten, um auf dieser Grundlage die Rolle der Medien zu beurteilen.

\section{Grundlagen der normalen Entwicklung}

Die zentrale Ausrichtung der frühen menschlichen Entwicklung auf psychosoziale Interaktion ist evolutionär zu begründen. So kommt der Mensch mit einem zur Endgröße relativ kleinen Kopf zur Welt, was durch das aus statischen Gründen kleine Becken zurückzuführen ist (aufrechter Gang). 70\% der Größenentwicklung des Gehirns (im Unterschied zu 30\% bei Schimpansen) finden beim Menschen aus diesem Grund extrauterin und somit unter dem starken Einfluss der Interaktion mit seiner Umgebung statt.

Merke

Die Interaktion stellt einen besonders differenzierten Stimulus dar, der für die Ausbildung eines komplexen neuronalen Netzwerks optimiert ist.

Durch diesen entscheidenden Einfluss auf die Gehirnentwicklung hat die frühe Interaktion des Säuglings mit seiner Umwelt in der Evolution der Menschheit eine besondere Bedeutung erlangt. In diesem Zusammenhang sind als bedeutsame Konzepte der frühen Interaktion etwa die intuitiven elterlichen Kompetenzen [3], die affektive Ab-

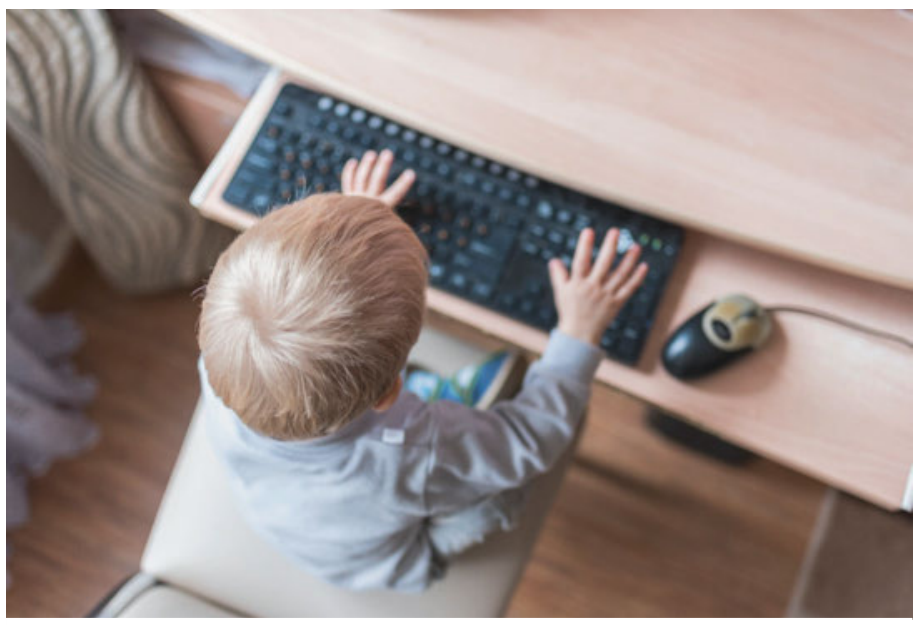

- Abb. 1 Bei der Frage nach dem Einfluss der neuen Medien auf die Kindergesundheit ist die Kindesentwicklung in den Blick zu nehmen (Symbolbild; Quelle: jalisko/Adobe Stock). 
stimmung („affect attunement“) zwischen bedeutsamer Bezugsperson und Säugling [4] und die soziale Rückversicherung („social referencing“) [5] zu nennen.

Die Interaktion stellt einen der wichtigsten und am stärksten abgesicherten Faktor für die Kindesentwicklung dar. Emotionale Bindung und emotionale Verfügbarkeit von Vertrauenspersonen sind dabei zentral. Mary Ainsworth [6,7] beschreibt die zentrale Rolle der Interaktion für die Kindesentwicklung in dem Modell der Feinfühligkeit vs. Unempfindlichkeit der Mutter bzw. Bezugsperson(en) gegenüber den Signalen des Babys (sensitivity vs. insensitivity to the baby's communication):

- Die Signale des Säuglings müssen richtig wahrgenommen werden (häufige Verfügbarkeit und niedrige Schwelle für kindliche Äußerungen),

- sodann richtig interpretiert werden (keine verzerrten Bewertungen, z.B. auf der Basis einer eigenen elterlichen Psychopathologie),

- und es muss in Folge prompt und angemessen darauf reagiert werden (z. B. geringe Latenzzeit der mütterlichen Reaktion, insbesondere auf Weinen und Quengeln des Säuglings).

Weitere, für Lernvorgänge während der frühen Kindheit förderliche Faktoren stellen die Variation und Variabilität von Bewegungsvorgängen dar, welche unterschiedliche sensorische Erfahrungen auslösen. Die dadurch getriggerten sensomotorischen Feedbackschleifen führen zur Etablierung neuronaler Netzwerke, in denen Bewegungsabläufe und Bewegungserfahrung hinterlegt werden. Von Relevanz sind kritische (im Gegensatz zu unkritischen bzw. beliebigen) Variationen von Bewegungsabläufen. Bei kritischen Variationen führen unter Umständen sehr geringe Veränderungen zu einem differenten Feedback. Der Säugling, der diskret in Richtung einer versehentlich liegengelassenen Schere blickt, wird (hoffentlich) bei seinen Eltern eine vollständig andere Reaktion auslösen, als wenn er - nur etwas die Richtung variierend - zu einem Spielzeug blickt.

Die Blickrichtung ist damit kritisch in Bezug auf das ausgelöste Feedback, eine für die Ausbildung neuronaler Strukturen wesentliche Voraussetzung. Kontraproduktiv hingegen wäre an dieser Stelle Beliebigkeit - die Blickrichtung des Säuglings ist unkritisch und löst entweder gar kein oder ein beliebiges, nicht differenzierendes Feedback aus. Begünstigend für den Lernvorgang ist hierbei die komplexe Ausprägung des Feedbacks. Zunächst löst jeder Bewegungsvorgang ein sensorisches Feedback durch Propriorezeption aus. Die Variation von Bewegungsvorgängen im dreidimensionalen Raum ist diesbezüglich als kritisch anzusehen, da sie zu einem differenten Feedback führt. Die Erweiterung des Feedbacks, z. B. der durch eine solche Bewegung entstehende Körperkontakt mit einer zusätzlichen sensorischen und emotionalen Komponente, erhöht die Komplexität der sensomotori- schen Feedbackschleife und der daraus resultierenden neuronalen Vernetzung.

\begin{abstract}
Merke
Auch wenn davon auszugehen ist, dass diese „Lernprinzipien“ lebenslänglich von Relevanz sind, spielen sie in den ersten 2 Lebensjahren in der Zeit der maximalen Gehirnentwicklung eine besonders hervorzuhebende Rolle.
\end{abstract}

Zum aktuellen Zeitpunkt liegen keine belastbaren Daten vor, die die Auswirkungen exzessiven elterlichen (v.a. mütterlichen) Medienkonsums auf die Interaktion, Bindung und Entwicklung des Säuglings aufzeigt. Unsere klinischen Beobachtungen auf zwei Eltern-Kind-Stationen (kbo-Kinderzentrum München und Eltern-Kind-Station der Kinder- und Jugendpsychiatrie Homburg/Saar) demonstrieren solche Auswirkungen in deutlichem Ausmaß.

\section{Einflüsse des Konsums moderner elektronischer Medien während der frühen Kindesentwicklung}

Kognition und Sprache

Bereits mit der Geburt zeigen Neugeborene eine Präferenz nicht nur für die Stimme der Mutter, sondern auch für die intrauterin wahrgenommene Muttersprache. Diese Reaktionsmuster auf die Muttersprache und auf Fremdsprachen unterscheiden sich zunehmend, auch wenn Kinder mit 6 Monaten noch eine 2. Sprache gleichwertig erlernen können.

\section{Merke \\ Aus EEG-Studien an Erwachsenen ist bekannt, dass akustische (und visuelle) Stimuli des Computerspiels vergleichbar sind mit suchtmittelspezifischen Reizen.}

Über den Prozess der klassischen Konditionierung werden zuvor neutrale Stimuli an das positive Erlebnis des Spielens gekoppelt und lösen danach die Erwartung eines positiven Erlebnisses aus. Exzessive Computerspieler zeigen daher - in Abgrenzung zu gelegentlich Spielenden - eine erhöhte EEG-Aktivierung bei Präsentation von Computerstimuli (erhöhte späte Positivierung als Maß der emotionalen Verarbeitungstiefe) [8]. Auf der Basis dieses Befundes kann spekuliert werden, in welchem Umfang etwa bei einer exzessiv computerspielenden Mutter schon in der Schwangerschaft auch die typischen auditiven Stimuli des Computerspiels vom Fetus intrauterin wahrgenommen und mit entsprechenden Gefühls- und Körperzuständen (z.B. Flow-Erlebnisse der Mutter) assoziiert werden können. Sind so auch Präferenzen für auditive Stimuli der Computerspiele schon beim Neugeborenen denkbar? 
Im Zusammenhang mit Multimediakonsum stellt sich die Frage, inwiefern Kinder sprachlich von Medien profitieren bzw. inwiefern sie konkret Sprache durch Medienkonsum erlernen können. Zunächst konnte gezeigt werden, dass 24 Monate alte Kinder einzelne Wörter durch interaktive Medienangebote lernen können. Entscheidend ist zunächst, dass dies nicht für alle, sondern nur für pädagogisch hochwertige Angebote gilt [9]. Darüber hinaus ist die Frage nach dem Vergleich mit anderen Sprachangeboten zu stellen: In einer Studie wurde die Reaktion von englischsprachigen Kindern auf die chinesische Sprache untersucht. Hierzu wurden 12 Spielsessions mit chinesisch sprechenden Kindern durchgeführt und in einer Vergleichsgruppe vergleichbare Bildschirmangebote vorgestellt. Die Reaktion auf die chinesische Sprache änderte sich in der Spielgruppe signifikant, nicht hingegen in der Bildschirmgruppe. Der direkte Kontakt und die Interaktion sind also beim Spracherwerb hervorzuheben. Ein weiterer, lerntheoretisch relevanter Aspekt stellt die direkte Alltagsanwendung dar, welche für den Spracherwerb von besonderer Relevanz ist. Die Kopplung von noch unbekannten Wörtern an direkt erlebte Situationen muss als entscheidender Faktor angesehen werden. Dies ist in der oben skizzierten Gruppensituation nicht nur durch die Interaktion (z.B. soziale Responsivität, Blickkontakt) gegeben, sondern darüber hinaus auch durch das Spielen, wodurch konkrete, aktiv durchgeführte Handlungen mit verbalen Äußerungen assoziiert werden.

\footnotetext{
Merke

Die Kopplung von direkter Handlungserfahrung mit Sprache kann über den Bildschirm nur eingeschränkt, im Vergleich zu der Spielsituation deutlich reduziert vermittelt werden.
}

PC-Spiele können sich negativ auf die kognitive Entwicklung und auf die Sprachentwicklung auswirken. In einer koreanischen Studie korrelierte der TV-Konsum negativ mit dem Erreichen der Schulreife (Review bei [9]). Eine japanische Studie zeigt einen Zusammenhang zwischen der Volumenreduktion der grauen und weißen Substanz und dem Fernsehkonsum ebenso wie mit dem Intelligenzquotienten [10].

Li u. Atkins [11] finden bereits im Vorschulalter positive Zusammenhänge von Computerspielen mit kognitiven Maßen. Dabei zeigte sich bei Vorschulkindern aus Familien mit niedrigem Einkommen, dass Kinder mit Zugang zu einem Computer besser bei Untersuchungen zur Schulreife abschnitten als Kinder ohne Computerzugang. Zudem zeigten die Kinder mit Computerzugang eine bessere Performanz bei Untersuchungen zur kognitiven Entwicklung - unter Kontrolle des kindlichen Entwicklungsstadiums und des sozioökonomischen Status der Eltern als die Kinder ohne Computerzugang. Gebrauch von und Umgang mit Computern bereits vor Schuleintritt scheinen also mit der Entwicklung von Vorschulkonzepten und kognitiven Prozessen assoziiert. Dabei scheint die Nutzungshäufigkeit jedoch keinen Einfluss zu haben. Analysen zum Zusammenhang zwischen Erfahrung mit dem Computer und visuomotorischen sowie grobmotorischen Fähigkeiten der Kinder zeigten keine signifikanten Ergebnisse. Das könnte jedoch, zumindest in Bezug auf die Visuomotorik, auch auf das verwendete Testverfahren zu deren Messung (Bender-Gestalt Test) [12] zurückzuführen sein, das laut den Autoren kein geeignetes Maß für die visuomotorische Entwicklung für Kinder dieser Altersgruppe bietet.

Einen weiteren gesicherten Einfluss hat die Anzahl der Wörter, die von Kindern zu Hause gehört werden. Sie korreliert nachgewiesenermaßen nicht nur mit dem sozioökonomischen Status, sondern auch mit dem Medienkonsum. In Familien, in denen tagsüber im Hintergrund der Fernseher läuft, wird signifikant weniger gesprochen, die Anzahl der Wörter, die während der Kindheit gehört wird, sinkt, was sich negativ auf den Spracherwerb auswirkt [9].

Zusammenfassend kann für den Spracherwerb festgehalten werden, dass das Erlernen einzelner Wörter zum Ende des 2. Lebensjahres durch pädagogische Bildschirmangebote erreicht werden kann - dieser Nachweis konnte allerdings nicht für ein jüngeres Lebensalter erbracht werden. Im 1. Lebensjahr konnte die Unterlegenheit von Medienangeboten für den Spracherwerb verglichen mit nicht digitalen Spielgruppenangeboten nachgewiesen werden. Aus lerntheoretischen Erwägungen muss von einer prinzipiellen Unterlegenheit von Multimediaangeboten verglichen mit unstrukturierten sozialen/interaktiven Spielen ausgegangen werden.

\section{Merke}

Ein übermäßiger Medienkonsum, z. B. der im Hintergrund laufende Fernseher, wirkt sich negativ auf die Sprachentwicklung eines Kindes aus.

\section{Motorische Entwicklung}

Gut belegt ist die Tatsache, dass sich körperliche Aktivität positiv auf die Gesundheit auswirkt [13]. Vor diesem Hintergrund empfiehlt die WHO für Kinder im Alter zwischen 5 und 17 Jahren täglich sportliche Betätigung von 1 Stunde Dauer, jeglicher darüber hinausgehenden sportlichen Aktivität wird ein zusätzlicher gesundheitlicher Nutzen zugeschrieben. Die als Messgröße für den Medienkonsum in Studien herangezogene „screen time“ korreliert negativ mit körperlicher Aktivität und der Ausprägung grobmotorischer Fähigkeiten und führt darüber hinaus zu einer erhöhten Inzidenz von Rücken- und Nackenschmerzen. Diese negativen Effekte des Medienkonsums lassen sich durch vermehrte sportliche Aktivität nicht ausgleichen [9]. 
Während es keine Hinweise aus Studien gibt, dass sich Medienkonsum positiv auf grobmotorische Fähigkeiten auswirkt, wird dies für feinmotorische Fähigkeiten diskutiert [14]. In einer retrospektiven Onlinebefragung von 366 Familien in Großbritannien mit Kindern im Alter von 19-36 Monaten wurde sowohl der Zeitpunkt des 1. Scrollens auf einem Touchscreens sowie das Erreichen von Meilensteinen der Entwicklung (Sprache, freies Sitzen und Laufen, Bauklötze aufeinanderstellen) erfasst. Während sich kein Zusammenhang zwischen grobmotorischen Fähigkeiten oder Sprache mit dem 1.Scrollen fand, korrelierte auch nach Korrektur für den mütterlichen Bildungsabschluss, Geschlecht und dem Alter des Erreichens des Pinzettengriffs das 1.Scrollen mit dem Aufeinanderstellen von Bauklötzen positiv. Es konnte ausgeschlossen werden, dass Kinder mit besseren feinmotorischen Fähigkeiten sowohl früher einen Touchscreen bedienen als auch Bauklötze aufeinander stellen (Korrektur für das Alter des Erreichens des Pinzettengriffs). Die Autoren diskutieren jedoch, inwiefern Kinder, die motiviert ihre Umgebung explorieren, dies in gleichem Maße mit einem Bildschirm und mit Bauklötzen machen oder ob von der Touchscreen-Benutzung mit entsprechendem Feedback ein positiver, stimulierender Effekt auf die feinmotorische Entwicklung ausgeht. Letzteres erscheint jedoch eher unwahrscheinlich, da die Nutzungsdauer des Touchscreens bei den Säuglingen nur bei durchschnittlich 8 Minuten pro Tag lag. Also muss in Frage gestellt werden, dass durch diese Studie eine positive oder zumindest „nicht negative“ Auswirkung der frühen Mediennutzung auf die Kindesentwicklung belegt wird.

\section{Merke \\ Hintergrund solcher Annahmen sind häufig Ergeb- nisse aus Erwachsenenstudien, die in das Kindes- und Säuglingsalter übertragen werden, ohne dass für diese Übertragbarkeit Evidenz besteht.}

Gezeigt werden konnte für Erwachsene, dass die visuellmotorischen Fähigkeiten bis hin zu Spezialistentätigkeiten (Flugmonitor, Laparoskopie) in Bildschirmsitzungen durch interaktive Mediennutzung sehr effektiv erlernt werden können.

Zusammenfassend kann festgehalten werden, dass eine negative Korrelation zwischen der Multimediaanwendung und körperlicher Aktivität besteht. Die Studienlage bezüglich der Auswirkung auf das Erlangen motorischer Fähigkeiten ist uneinheitlich. Belastbare Belege dafür, dass Multimedia-Spielangebote bezüglich der Interaktion, der Dreidimensionalität, der kritischen Variabilität des Outputs und des differenzierten spezifischen Feedbacks eine vergleichbare Qualität wie nicht digitale Spielangebote haben, existieren nicht. Entsprechende Behauptungen sollten also kritisch hinterfragt werden.

\section{Psychosoziale Entwicklung}

Durch Studien wird ein Zusammenhang zwischen übermäßigem Medienkonsum und psychischen Erkrankungen belegt. So konnte gezeigt werden, dass Fernsehkonsum im Alter von 6-18 Monaten mit dem Auftreten von Aggressivität und externalisierenden Verhaltensstörungen zusammenhängt. Für Jugendliche konnte eine negative Auswirkung von Fernsehkonsum auf die seelische Gesundheit und auf die schulische Leistungsfähigkeit nachgewiesen werden. In einer Kohorte von 2500 kanadischen Jugendlichen im Alter von 12-18 Jahren konnte eine Korrelation der Screen Time mit der Ausprägung von Angststörungen und Depression vor allem für digitale Spiele nachgewiesen werden [15].

Umgekehrt konnte für 8000 australische Kinder und Jugendliche im Alter von 10-18 Jahren gezeigt werden, dass diejenigen, die die Empfehlung, 1 Stunde Screen Time pro Tag nicht zu überschreiten, beherzigt hatten, signifikant weniger von Depressionen betroffen waren [16]. Ein Zusammenhang konnte des Weiteren zwischen Fernsehkonsum im Alter von 1-2 Jahren und Aufmerksamkeitsstörungen im Alter von 7 Jahren nachgewiesen werden. Eine 2. Studie schränkt dies auf den Konsum von nicht pädagogischen Angeboten ein, für pädagogische Angebote hingegen konnte dieser Effekt nicht nachgewiesen werden. Bezüglich der sozialen Auswirkungen konnte gezeigt werden, dass Multimediaspiele, welche konkret soziale Interaktion zum Gegenstand haben, diese verbessern können. Übermäßiger Fernsehkonsum reduziert die innerfamiliäre Kommunikation, erhöht das Risiko für Mobbing durch Gleichaltrige, darüber hinaus führt der verstärkte Medienkonsum der Eltern zu einer reduzierten Eltern-Kind-Interaktion und erhöht das Risiko für Konflikte [17]. In den sog. sozialen Medien („social media“) wird dagegen die Möglichkeit eines Kommunikationskanals mit Gleichaltrigen und geografisch entfernten Verwandten gesehen [18].

\footnotetext{
Merke

Die Zusammenhänge zwischen einem übermäßigen Fernsehkonsum und dem Auftreten psychischer Erkrankungen sind gut belegt.
}

Deutlich schwächere Hinweise bestehen für das Auftreten psychischer Erkrankungen wie Depression und Aufmerksamkeitsstörungen im Zusammenhang mit einem erhöhten und sehr frühen Konsum von Computer- und Videospielen. Das Einhalten der Empfehlungen der Australischen Pädiatrischen Fachgesellschaft (Screen Time im Alter unter 2 Jahren gleich 0 , über 2 Jahren maximal 1 Stunde pro Tag) korrelieren mit einer geringeren Inzidenz von Depressionen bei Jugendlichen. 


\section{Ernährung und Schlaf}

Die Mediennutzung beeinflusst das Ess- und Schlafverhalten von Kindern und Jugendlichen. Dieser Effekt zeigt sich besonders bei kleinen Kindern. In einer Studie an 1000 Kindern im Alter von 2-6 Jahren führte bereits 1 Stunde Fernsehkonsum pro Tag zu einer signifikanten Schlafreduktion. Bei 11- bis 15-jährigen Kindern hingegen wurde die Schlafdauer nicht durch einen erhöhten Fernsehkonsum (3 Stunden pro Tag), jedoch durch einen entsprechenden Video-/PC-Spiele-Konsum beeinträchtigt [19].

Massive Effekte von Fernsehkonsum auf die Ernährung sind in mehreren Studien belegt. So korreliert eine Dauer von 2 und mehr Stunden Fernsehen pro Tag mit Fettleibigkeit (NHANES-Studie), eine Metaanalyse von 14 Studien belegt, dass bereits 1 Stunde Fernsehen pro Tag das Risiko für Adipositas um 13\% erhöht [20]. Darüber hinaus konnte eine Untersuchung an 10000 Kindern (6-9 Jahre alt) belegen, dass jede zusätzliche Stunde Screen Time den Konsum von hoch zucker- und fetthaltigen Produkten (Limonade, Energydrink, Schokolade, Hamburger) erhöht und den Konsum von Gemüse und Obst verringert Effekte, die durch die automatische Exposition gegenüber den eingeblendeten Werbesendungen zusätzlich verstärkt werden.

\section{Konsumverhalten: aktuelle Datenlage zum Medienumgang}

Befragungen zum Thema Medienkonsum unterliegen möglicherweise einem Bias im Sinne eines Trends hin zu sozial erwünschten Angaben.

\section{Merke}

Damit besteht ein erhebliches Risiko, dass Medienkonsum eher unter- als überschätzt wird.

In der repräsentativen MiniKIM-Studie 2014 [21] wurden 600 Haupterzieher (Elternperspektive, zumeist Mütter) in Deutschland zur Mediennutzung ihrer 2- bis 5-jährigen Kinder (die selbst noch keine ausreichende Auskunftsfähigkeit haben) befragt und mit den Ergebnissen von 2012 [22] verglichen. Ausgewählte Ergebnisse der MiniKIM-Studie sind in der Infobox zusammengefasst und kommentiert.

\section{Exzessive Mediennutzung}

\section{Computerspiel- und Internetabhängigkeit}

Computerspiel- und Internetabhängigkeit ist zum aktuellen Zeitpunkt keine nosologische Entität nach ICD-10, dies kontrastiert mit dem klinischen Alltagseindruck bei manchen Patientenvorstellungen. Im DSM-5 erfolgte 2013 zum ersten Mal eine Nennung dieses Störungsbil-
INFO

Ausgewählte Ergebnisse der MiniKIM-Studie (2014) [21]

Medienverfügbarkeit

- (nahezu) Vollausstattung von Haushalten, in denen 2- bis 5-Jährige leben, für Fernseher, Handy/Smartphone und Computer/Laptop

- $8 \%$ der Kinder verfügen über Spielkonsole; deutliche Altersabhängigkeit (3\% bei 2- bis 3-Jährigen; $13 \%$ bei 4- bis 5-Jährigen), keine bedeutsamen Geschlechtsunterschiede

- über Handy/Smartphone verfügen 2\% aller 2- bis 5-jährigen

\section{Hauptaktivitäten}

- aktiv genutztes Medienrepertoire steigt zwischen 2 und 5 Jahren stark an, insbesondere beim Fernsehen und bei Computer-/ Konsolen-/Online-Spielen

- 1/4 der 4- bis 5-Jährigen nutzt digitale Spiele

- mit dem Internet haben 7\% Erfahrungen gemacht, 5\% nutzen das Internet regelmäßig

- 1. Internetnutzung im Schnitt mit 3,8 Jahren

- $86 \%$ der Eltern stimmen der Meinung, „das Internet ist für Kinder gefährlich“, voll und ganz bzw. überwiegend zu (3\% weniger als 2012), 13\% erlauben ihrem Kind, ohne Aufsicht im Internet zu surfen (3\% mehr als 2012)

\section{Nutzungsdauer und -kontext}

- Mädchen und Jungen schauen durchschnittlich 43 min proTag Fernsehen (im Alter 2-3 Jahre: 34 min; im Alter 4-5 Jahre: $52 \mathrm{~min})$

- PC-/Online-/Konsolenspiele werden 3 min gespielt (1 min bei 2- bis 3-Jährigen; 5 min bei 4- bis 5-Jährigen) - gemittelt über alle Nutzer (aber: geringe Nutzerzahl, daher: wesentlich längere Nutzungsdauer bei Kindern mit Gerätebesitz)

\section{Kindertagesstätten}

- 13 \% der Kindergärten/Krippen verfügen über Computer (11\% im Jahr 2012)

- $7 \%$ über Internet (2\% im Jahr 2012).

- in $4 \%$ der Kindergärten oder Krippen stehen den Kindern im Jahr 2014 Tablets zur Verfügung

- Studien zu medienbezogenen Tätigkeiten, zu Medienbindung und Nutzungsdauer im Kindergarten fehlen derzeit

- Zentral für die Kompetenzentwicklung: Vorlesen [23]

des als „Störung durch Spielen von Internetspielen“ („Internet Gaming Disorder“), allerdings nur unter der Rubrik „Klinische Erscheinungsbilder mit weiterem Forschungsbedarf“, sozusagen als Forschungsdiagnose, nicht aber als klinische Diagnose.

Dennoch ist diese erste Nennung in einem Diagnosemanual als „Meilenstein“ zu bewerten. Es handelt sich nach DSM-5 um eine dauerhafte Nutzung des Internets, um sich mit Spielen zu beschäftigen, die zu klinisch bedeut- 
INFO

\section{Kriterien der Internet Gaming Disorder (nach DSM-5, S. 1088 f.)}

- übermäßige Beschäftigung mit Internetspielen (an vorherige oder zukünftige Spielaktivitäten, Absicht, das nächste Spiel zu spielen, Spielen von Internetspielen wird zur Haupttätigkeit des Tages)

- Entzugssymptomatik, wenn das Spielen von Internetspielen wegfällt (typischerweise Symptome von Reizbarkeit, Ängstlichkeit, Traurigkeit, jedoch keine körperlichen Zeichen eines pharmakologischen Entzugssymptoms)

- Toleranzentwicklung (Bedürfnis, zunehmend mehr Zeit mit dem Spielen von Internetspielen zu verbringen)

- Interessensverlust an früheren Hobbys und Freizeitbeschäftigungen als Ergebnis und mit Ausnahme des Spielens von Internetspielen

- erfolglose Versuche, die Teilnahme an Internetspielen zu kontrollieren

- exzessives Spielen von Internetspielen trotz der Einsicht in die psychosozialen Folgen

- Täuschen von Familienangehörigen, Therapeuten und anderen bezüglich des Umfangs des Spielens

- Nutzen von Internetspielen, um einer negativen Stimmungslage zu entfliehen oder sie abzuschwächen (z. B. Gefühl der Hilflosigkeit, Schuldgefühle, Ängstlichkeit)

- Gefährdung oder Verlust einer wichtigen Beziehung, der Arbeitsstelle oder Ausbildungs-/Karrieremöglichkeit aufgrund der Teilnahme an Internetspielen

samen Beeinträchtigungen oder Leiden führt und für die mindestens 5 der 9 Kriterien aus der Infobox in einem Zeitraum von 12 Monaten vorliegen müssen. Für das Spielverhalten im Vorschulalter (und teilweise in der weiteren Kindheit) ist die Charakterisierung „Internetspiele“ zu ersetzen durch „Computerspiele“, da in dieser Altersgruppe das Spielen mit stationären oder transportablen Spielekonsolen gegenüber internetbasierten Spielen dominiert. In der Betaversion des ICD-11 findet sich „Gaming Disorder“ als eigenständige nosologische Entität [24].

„Neben dem Positiven, die das Anwenden und Verstehen dieser modernen Technologie und der technischen Geräte mit sich bringt, entfalten sich gefährliche Wirkungen für Kinder und Jugendliche, wenn

- aus der exzessiven Nutzung sekundär körperliche oder psychische Probleme oder gar Störungen entstehen,

- auf der Basis prämorbid bestehender psychischer Störungen die exzessive Computer- und Internetnutzung zum (dysfunktionalen) Lösungsansatz oder zur aufrechterhaltenden Bedingung für ebendiese psychischen Störungen wird,

- aufgrund exzessiver Nutzung das Ausüben anderer Lebensaktivitäten (z. B. Schule, Wissenserwerb, Ausbildung, Sport, Beteiligung im Verein) vernachlässigt oder gänzlich aufgegeben wird,
- die exzessive Nutzung die weitere bio-psycho-soziale Entwicklung des Kindes oder Jugendlichen beeinträchtigt, so dass entwicklungsphasentypische Aufgaben nicht bewältigt und Kompetenzen nicht erworben werden können“" [25].

\section{Merke}

Ein falscher Einsatz und ein zu früher und unbegrenzter Konsum von Computerspielen können einen negativen Einfluss auf die psychische und körperliche Gesundheit von Kindern haben.

Mit hoher Wahrscheinlichkeit sind die Ergebnisse bezüglich Bewegungsarmut und Ernährungsgewohnheiten auf die Nutzung von Multimedien übertragbar. Negative Auswirkungen auf die psychische Gesundheit konnten speziell für gewalthaltige PC- und Videospiele nachgewiesen werden.

Häufiges Spielen gewalttätiger Computerspiele steht bei Kindern und Jugendlichen in Zusammenhang mit einer Zunahme von aggressiven Verhaltensweisen, Gedanken und Gefühlen, von emotionaler Abstumpfung gegenüber Gewalt, von vegetativer Erregtheit, abnehmender Hilfsbereitschaft und einem Mangel an Empathie [26-30]. Die Stärke der kindlichen Aggression wird mit der Häufigkeit und der Dauer des Konsums gewalthaltiger Medien assoziiert. Einige experimentelle Studien konnten hierbei nicht nur einen korrelativen, sondern einen kausalen Zusammenhang nachweisen $[28,29]$.

Darüber hinaus haben Schüler, die regelmäßig Computerspiele mit gewalttätigen Inhalten spielen, öfter Auseinandersetzungen mit Lehrern und Gleichaltrigen und häufiger Leistungsprobleme in der Schule [31]. In methodisch hochwertigen Studien finden sich zudem größere Effekte als in Untersuchungen mit methodischen Mängeln, was vermuten lässt, dass die negativen Konsequenzen gewalttätiger Spiele sogar größer sind als ursprünglich angenommen [29]. Allerdings beschränken sich viele Untersuchungen zu den negativen Auswirkungen von Computerspielen auf Computerspiele mit gewalttätigen Inhalten.

Weiterhin konnten Untersuchungen zeigen, dass sich die Nutzung moderner Medien auf die Schlafdauer und Schlafqualität auswirken kann [32]. So zeigten Strube et al. [33] einen Zusammenhang zwischen einer häufigen Nutzung und dem Umgang Smartphones mit der Tagesschläfrigkeit von Jugendlichen auf. Zudem kann Videospielen vor dem Zubettgehen die Einschlaflatenz verlängern und die Schläfrigkeit von Jugendlichen erhöhen [32]. Auch wenn die Nutzung von Smartphones im Vorschulalter noch deutlich weniger verbreitet ist als bei Jugendlichen, wäre eine Untersuchung der Auswirkung moderner Medien auf das Schlafverhalten von Kindern im Vorschulalter wichtig. 
Wie auch in Bezug auf den Fernsehkonsum lässt sich ein Zusammenhang zwischen Adipositas und Computernutzung im Vorschulalter auffinden [34].

Allerdings zweifeln einige Autoren eine ausreichende wissenschaftliche Befundlage an, um gesicherte Aussagen über die Auswirkungen häufigen Spielens auf die Gesundheit und das Verhalten von Kindern treffen zu können (z.B. [35-37]). Appel u. Schreiner [38] argumentieren, dass die Vermischung von korrelativen Zusammenhängen und Kausalität oder die selektive Berücksichtigung von Einzelstudien hierbei zu den bedeutendsten Fehlerquellen gehören.

\section{Neurobiologische Konsequenzen exzessiven Computerspielens}

Häufiges Computer- oder Konsolenspielen steht nicht nur mit Veränderungen auf der Verhaltensebene in Zusammenhang, sondern auch mit neurobiologischen Anomalien. Dabei fanden Studien z. B. Veränderungen des Volumens der grauen Substanz, Störungen der funktionellen Konnektivität und der Aktivierung spezifischer Hirnregionen und neurobiologische Zusammenhänge mit störungsspezifischen Verhaltensweisen und Symptomen [39].

\section{Merke \\ Bisher ist jedoch unklar, ob diese neurobiologischen Anomalien Ursache oder Auswirkung des häufigen Spielekonsums sind.}

In vorherigen Studien konnten einige verhaltensbezogene und neurobiologische Gemeinsamkeiten zwischen pathologischem Spielen und Abhängigkeitserkrankungen wie Substanzabhängigkeit gefunden werden [40]. So konnte das impulsive Verhalten Betroffener mit defizitären frontostriatalen Netzwerken, Störungen der interhemisphärischen Konnektivität und einer veränderten Struktur der Inselrinde assoziiert werden [41, 42]. Störungen der Regulation des emotionsgetriebenen Verlangens könnten auf Funktionsstörungen des präfrontalen Kortex zurückzuführen sein $[41,43]$.

Weiterhin wiesen Betroffene ein beeinträchtigtes Belohnungssystem auf [44-47]. Dabei spielt der mesolimbische Dopaminpfad eine wichtige Rolle. Untersuchungen konnten zeigen, dass ein exzessiver Spielekonsum mit einem generellen Belohnungsdefizit assoziiert ist, welches mit einer reduzierten dopaminergen Aktivität in Zusammenhang steht [48]. Ein zu niedriger Dopaminlevel wird mit Impulsivität und riskanten Entscheidungen assoziiert [49]. Da Videospielen eine Dopaminausschüttung auslöst [48], wird so vermutlich der Dopaminmangel (kurzfristig) ausgeglichen.

\section{Nutzen und Schaden elektronischer Medien}

Eine medienfreie Kindheit mag es im Einzelfall noch als theoretisches Idealkonzept des Bewahrens (,früher war alles besser“) geben, was aber mit der Realität der Kinderwelt, der Familie und dem Kindergarten keine Passung zeigt. Sinnvoll scheint es zu sein, zwischen funktionalem und dysfunktionalem Mediengebrauch zu unterscheiden und dies entwicklungsaltersangemessen einzuordnen. Auch wenn ein übermäßiger und unkritischer Medienkonsum erhebliche gesundheitliche Risiken für Kinder und Jugendliche birgt und manche aktuellen Nutzungsgewohnheiten als gesundheitsgefährdend eingestuft werden müssen, werden extern oktroyierte Vorschriften und Regeln wohl wenig Erfolg im Sinne von Verhaltensveränderungen bewirken.

So kommt eine Untersuchung zu dem Ergebnis, dass die Empfehlungen der amerikanischen oder australischen pädiatrischen Fachgesellschaften von mindestens $2 / 3$ der Familien nicht umgesetzt werden [50]. Von Interesse sind auch die Gründe, die Eltern dafür angeben, dass ihre Kinder Medien nutzen. In einer amerikanischen Studie wird von Eltern als häufigster Grund „it is good for their brains" angegeben [2]. Als weitere Gründe sind Ablenkung und Beschäftigung (z. B. während die Eltern arbeiten/den Haushalt erledigen) und Vermeidung von Konflikten durch das Durchsetzen von Regeln im Umgang mit Medien genannt worden. Als weiterer Faktor wurden finanzielle Gründe angegeben, wonach schlicht das Geld für andere Freizeitaktivitäten fehlen würde.

\section{Differenzierte Betrachtung erforderlich}

Bei der Beurteilung von positiven und negativen Effekten von Mediennutzung auf die Kindergesundheit muss zwischen der Art der Medien und dem Alter differenziert werden. Grundsätzlich sind die Auswirkungen von Fernsehkonsum besser erforscht als die von anderen Medien.

\footnotetext{
Merke

Zahlreiche Studien belegen, dass die Qualität der Angebote von Relevanz ist.
}

Zu unterscheiden ist zwischen pädagogischen hochwertigen und nicht pädagogischen Angeboten, wobei Angaben und Studien hierbei kritisch auf die Beeinflussung durch Herstellerinteressen hinterfragt werden müssen. Gesichert ist auch die Erkenntnis, dass Ergebnisse aus dem Erwachsenenalter nicht in das Kindesalter übertragen werden können - denn bereits die Effekte der Mediennutzung differieren in den ersten 2 Lebensjahren und im Vorschulalter stark. Entwicklungsneurologische und bindungs- und interaktionszentrierte Aspekte, Entwicklungsaufgaben und sich entwickelnde Beeinträchti- 
gungen (impairment) sind bei der Beurteilung der Auswirkungen von Medienkonsum von hoher Relevanz.

Die kindliche Entwicklung allgemein und die Entwicklung psychischer Störungen im Kindesalter im Besonderen sind immer zu betrachten auf der Basis der Bilanzierung von Belastungen und Risikofaktoren (z.B. Disposition und Vulnerabilität des Kindes; umgebungsbezogene Stressoren) im Abgleich mit Schutzfaktoren und Ressourcen (z. B. Resilienz des Kindes, soziale Unterstützung der Umgebung). Diese komplexe Dynamik von Risiko- und Schutzfaktoren der kindlichen Entwicklung bildet den Hintergrund, in dem die Mediennutzung ihre Auswirkungen entfaltet.

\section{Merke}

Nicht zuletzt unterliegt Medienkonsum stark sozialen Einflüssen, die bei der Beurteilung der Auswirkung auf die Gesundheit als Einflussvariablen zu berücksichtigen sind.

\section{Fernsehkonsum - möglicher Benefit}

Filme zur Wissensvermittlung sind aus E-Learning-Konzepten der Erwachsenenbildung nicht wegzudenken. Übertragbar ist dies auf Jugendliche und Schulkinder weitgehend, auf Vorschulkinder nur sehr eingeschränkt und keinesfalls auf die ersten 2 Lebensjahre. Die Studienlage hierfür lässt sich am Beispiel der „Sesamstraße“, der am besten evaluierten Kinderfernsehsendung überhaupt, zusammenfassen: Die als pädagogisch und didaktisch hochwertig international anerkannte Sendung zeigt positive Effekte für Kinder ab dem 3. bis zum 5. Lebensjahr, bei jüngeren Kindern wurden sogar negative Effekte beobachtet.

\section{Merke}

Kinder im 1. und 2. Lebensjahr haben Schwierigkeiten, Informationen von der zweidimensionalen Bildschirmebene in die dreidimensionale Realität zu übertragen und profitieren von der Interaktion mit der Umgebung und der direkten Erfahrung mit den Händen.

Auch gelang es bisher nicht, positive Effekte für den Spracherwerb durch Bildschirmangebote nachzuweisen, diese sind erstmals zum Ende des 2. Lebensjahres zu beobachten. Neben möglichen negativen Effekten ist auch zu berücksichtigen, inwiefern Wissensvermittlung nicht durch nicht digitale Angebote, welche interaktiv, dreidimensional und eigene Handlungserfahrungen offerieren, besser und effektiver erfolgen kann. Das gemeinsame Anschauen einer Fernsehsendung von Eltern und Kind integriert zumindest teilweise eine interaktive Komponente.

\section{Fernsehkonsum - negative Auswirkungen}

Negative Auswirkungen von übermäßigem Fernsehkonsum auf die psychische und körperliche Gesundheit sowie auf die motorische, kognitive und sprachliche Entwicklung sind umfassend belegt. Fernsehkonsum in der frühen Kindheit korreliert mit Aufmerksamkeitsstörungen im 7. Lebensjahr und Fernsehkonsum im Schulalter mit depressiven Störungen. Auch exekutive Funktionen können im Kindesalter durch das Anschauen von Fantasiefilmen beeinträchtigt werden [51]. Bereits 1 Stunde Fernsehen täglich erhöht das Risiko für Adipositas, es besteht eine enge Beziehung zwischen Fernsehkonsum und zuckerhaltiger und fettreicher Ernährung. Hervorzuheben ist, dass diese Studienergebnisse für die Einflussfaktoren Bildung bzw. sozioökonomischer Status korrigiert sind. Erhöhter Fernsehkonsum erhöht das Risiko für Entwicklungsverzögerungen erheblich und führt zur Verzögerung der Schulreife.

Auch wenn die meisten negativen Auswirkungen erst durch einen hohen Fernsehkonsum (2 Stunden und mehr pro Tag) entstehen, ist zu bedenken, dass dieser dem durchschnittlichen Nutzungsverhalten durchaus nahe kommt. Dies wirft die Frage nach der generellen Akzeptanz des Mediums Fernsehen insbesondere für das Kindesalter auf. Das ausgeweitete und vervielfältigte Angebot von Sendungen und Formaten im Kinderfernsehen ist vor diesem Hintergrund folglich kritisch zu hinterfragen, hat es doch erheblich dazu beigetragen, dass die Nutzung des Fernsehens auch bei Kleinkindern stetig zunimmt. Die negativen Effekte des Fernsehkonsums werden aber durch pädagogisch hochwertige Angebote nicht aufgewogen.

\section{Spiele und interaktive Mediennutzung - Benefit}

Wie bei der Wissensvermittlung durch Fernsehen hat auch die interaktive Mediennutzung ihren festen Platz in der Erwachsenenbildung, besonders Spezialkompetenzen können mit computergestützten Simulatoren sehr effektiv erlernt werden. Dies ist nur sehr eingeschränkt in das Jugend- und Schulalter zu übertragen, im Kleinkindalter und für das Säuglingsalter gibt es keine Hinweise, dass Wissenstransfer auf diese Art und Weise effektiv erfolgen kann.

Computerspiele ohne gewalttätige Inhalte zeigen einen positiven Effekt auf das räumliche Vorstellungsvermögen und die Verarbeitung visueller Informationen bei Kindern und Jugendlichen [52,53]. Li u. Atkins [11] finden bereits im Vorschulalter positive Zusammenhänge von Computerspielen mit der kognitiven Entwicklung. Daher ist es naheliegend anzunehmen, dass spezifische Computerspiele bei altersangemessener Anwendung und entsprechender Indikation auch in der Vorschule und Schule einen Zugewinn bringen können. 
"Spielerisch“ kann beispielsweise das gesundheitsbezogene Verhalten von Kindern gesteigert werden [54]. Neben der Linderung spezifischer Symptome und Schmerzen bei krebskranken Kindern werden sie auch unterstützend bei psychischen Erkrankungen wie LRS oder ADHS eingesetzt, um Lernprozesse zu festigen [55 - 57]. Die Indikation von digitalen Medien zum therapeutischen Einsatz in der täglichen Praxis ist jedoch stets in jedem Einzelfall kritisch zu hinterfragen, d.h. ein Angehöriger welcher Berufsgruppe mit welcher Zusatzausbildung wendet zum Erreichen welchen Behandlungszieles welche spezifischen computergestützten Methoden an?

Merke

Ein unkritischer, unspezifischer Einsatz ohne klare Indikation, ohne differenzierte Behandlungsplanung, ohne randomisiert-kontrollierten Wirksamkeitsnachweis der Methode und ohne individuelle Evaluation ist strikt abzulehnen.

\section{Handlungsempfehlungen}

\section{Beratung: Definition von Entwicklungszielen zur Entwicklungsförderung}

Eine effektive Beratung setzt bei der gemeinsamen Erarbeitung von Entwicklungszielen an. Die Grundhaltung „Bis das Gegenteil bewiesen ist, gehen wir davon aus, dass Eltern für ihr Kind das Bestmögliche erreichen wollen“, stellt hier den Ausgangspunkt dar und „Was wollen wir für das Kind erreichen, was wünschen wir uns für unser Kind?“, ist die gemeinsame Gesprächsbasis. In der Regel werden in diesem Zusammenhang „glücklich sein“, Gesundheit und ein „normales“ selbstständiges Leben als Wünsche für die Zukunft angegeben.

Die zentrale Bedeutung der Interaktion zwischen Eltern und Kind, die emotionale Verfügbarkeit von Eltern und die emotionale Bindung zwischen Eltern und Kind sind hierbei in wissenschaftlichen Studien als entscheidende Faktoren vor allem in der frühen Kindheit identifiziert worden, um genau diese von den Eltern formulierten Ziele zu erreichen.

\footnotetext{
Merke

Die Beschäftigung mit Kleinkindern muss sich also daran orientieren, wie sie dieser Interaktion am besten zuträglich ist, andere Ziele müssen sich dem zunächst unterordnen.
}

Hervorzuheben ist, dass diese Priorisierung keinen eigenen Regelkatalog per se darstellt. Vielmehr geht es darum, das Vertrauen in die eigene elterliche Intuition für „das, was für das eigene Kind gut ist“, zu stärken und in das Zentrum des eigenen Handelns zu stellen. Damit wird vermieden, dass konkurrierende und widersprüchliche
Handlungsempfehlungen zu Gleichgültigkeit und Passivität führen:

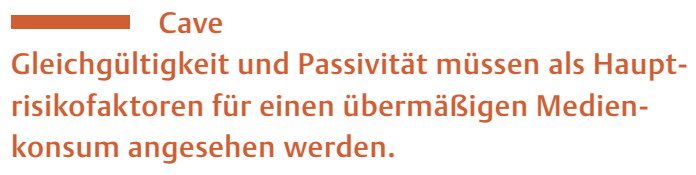

In Abhängigkeit vom Alter des Kindes und vom Beratungsbedarf der Eltern können Entwicklungsziele, z.B. für einen Schulerfolg, präzisiert werden. Von besonderer Relevanz sind Fähigkeiten wie Task Persistence, Impulskontrolle, emotionale Regulation, soziale Kompetenz und kreatives und flexibles Denken.

Merke
Wichtig ist es hervorzuheben, dass der routinierte
Umgang mit Medien per se für den Schulerfolg keine
Voraussetzung darstellt und vielmehr davon aus-
gegangen werden kann, dass er unkompliziert
„nebenbei“ erlernt werden kann.

\section{Empfehlungen für Kinder- und Jugendmedizin,} -psychiatrie und -psychotherapie

Empfohlen wird routinemäßig ein Screening und bei Verdachtsmomenten eine detaillierte Anamnese der Medienverfügbarkeit und der Nutzungsbedingungen bei Kindern und deren Eltern, ggf. auch der Kriterien für eine Computerspielabhängigkeit [25, 58, 59]. Ergänzend sollte ein Screening möglicher komorbider psychischer Störungen erfolgen, insbesondere für ADHS/HKS, Depression, aber auch für soziale Phobie und Autismus-SpektrumStörungen als häufige komorbide Störungen [59,60], und das auch schon im Vorschul- und Einschulungsalter [61].

Im Rahmen der Psychoedukation können den Eltern für den Altersbereich der frühen Kindheit Informationen über die Grundsätze der Gehirnentwicklung, der Bindungsentwicklung und der elterlichen Feinfühligkeit vermittelt werden, ebenso wie die möglichen Einflüsse exzessiven Medienkonsums. Insbesondere sollte die Bedeutung von unstrukturierten „Hands-on“-Tätigkeiten und die Bedeutung sozialer Interaktionen und Spielen zur Sprachförderung und Entwicklung von sozioemotionalen Skills hervorgehoben werden.

\footnotetext{
Merke

Unbedingt sollte der Hinweis erfolgen, dass übermäßige (und unbegleitete) Mediennutzung gerade für sehr kleine Kinder sehr ungünstig ist und - wenngleich bequem im Sinne von Ruhigstellen - Vernachlässigungsaspekte aufweisen kann.
}

Der familiäre Medienkonsum bzw. Nutzergewohnheiten (Art der Medien, Nutzungsorte, Nutzungsdauer) sollten bereits früh, im Säuglingsalter, thematisiert werden. 
Schon Säuglinge zeigen Orientierungsreaktionen zu Bildschirmen (Fernseher, Computer) im Sinne des Hinwendens des Kopfes zu Reizquellen, die Aufmerksamkeit erzeugen. Töne wecken vermutlich in noch stärkerem Maße das Interesse im Säuglings- und Kleinkindalter. In diesem Rahmen sind frühe Lernprozesse (klassische und operante Konditionierung) anzunehmen.

Schon Kleinkinder ahmen im Sinne des Modelllernens ihre Eltern nach. Über gemeinsame Aufmerksamkeit (,joint attention") kann Aufmerksamkeit nicht nur für ein Kinderbuch, sondern auch für Filme mit gewalthaltigen oder pornografischen Inhalten hergestellt werden, wenn dies auch nicht automatisch ein entsprechendes inhaltliches Interesse des Kindes bedeutet. Dagegen sind diese Medienerfahrungen von Säuglingen den Eltern allgemein nicht bewusst; deren Auswirkungen sind weitgehend ungeklärt.

Mit den Eltern sollten Probleme bei der Grenzsetzung angesprochen werden, sie sollten hinsichtlich des Entwickelns von Alternativangeboten unterstützt werden. Ein eigenständiger Medienumgang mit Computer und Internet, aber auch mit Fernsehen vor der Einschulung scheint nicht nützlich, eher eine eingeschränkte Nutzung mit elterlicher Unterstützung.

Dringend abzuraten ist von WLAN-fähigem Spielzeug, das aus dem Kinderzimmer filmen oder das kindliche Spiel aufnehmen und zum Abhören durch die Eltern speichern kann („Smart Toys“). So wurde die Kinderpuppe „My Friend Cayla“ im Februar 2017 von der Bundesnetzagentur vom Markt genommen, da sie eine unerlaubte funkfähige Sendeanlage darstellt [62], die auch gehackt werden kann. Smart Toys berühren neben juristischen Fragen insbesondere auch die ethische Fragestellung, ob das Kind ein Recht auf Privatsphäre auch gegenüber den eigenen Eltern hat. Sie ermöglichen aber nicht nur eine Speicherung und nachträgliche Wiedergabe des Spiels ihres Kindes mit der Puppe durch die Eltern, sondern erlauben bei Sicherheitslücken auch Fremden, sich auf diesem Kanal Zugang zum Kinderzimmer und zum Kind zu verschaffen (sodass das Kind meint, die Puppe spreche mit und zu ihm). Zusätzlich können sie teilweise auch der ökonomisch verwertbaren Bedürfniserzeugung bei kleinen Kindern dienen, wenn bestimmte Produkte durch die Puppe als Sendeanlage in der kindlichen Welt platziert werden [63].

\section{Empfehlungen an die Eltern}

Eltern nehmen die Position als Modell der Mediennutzung (Dauer, Nutzungsstil) für die Kinder und gleichermaßen erzieherisch im Aufstellen und nachhaltigen Durchsetzen von Mediennutzungsregeln ein. Eltern legen (bewusst oder unreflektiert) fest, mit welchen Medien in welchem Ausmaß und unter welchen Rahmenbedingungen (beiläufig unkontrolliert, kontrolliert und gezielt, be- gleitet oder allein) das Kind seine eigenen Medienkontakte hat.

\section{Merke \\ Das Elternhaus ist der zentrale Ort für die frühe Mediensozialisation der Kinder.}

Vorschulkinder sind wegen des Entwicklungsstandes nicht medienmündig im Sinne einer überdachten und selbstbestimmten Nutzung. Risiken lassen sich umso mehr reduzieren, indem der Medienkonsum durch die Eltern geringgehalten und eher später ermöglicht wird [64]. Vom Medienkonsum zu unterscheiden ist das begleitete Erkunden der digitalen Medien und deren Anwendung mit alltagsrelevanten Zielsetzungen.

In den USA wird von der amerikanischen Fachgesellschaft der Kinderärzte von einer „gesunden Medien-Diät“ gesprochen und die Erstellung von „Mediennutzungsplänen“ empfohlen (www.healthychildren.org/MediaUsePlan):

- Für Kinder unter 18 Monaten sollte Medienerfahrung grundsätzlich vermieden werden.

- Wenn die Einführung von Medien von den Eltern gewünscht wird, dann können bei Kindern im Alter von 18-24 Monaten pädagogisch hochwertige Medien unter Begleitung/Anwesenheit einer Bezugsperson eingesetzt werden. Selbstständige Mediennutzung sollte in diesem Alter strikt vermieden werden, eine Verwendung zur Ablenkung der Kinder oder als Quasi-Babysitter ebenfalls.

- Bei Kindern im Alter zwischen 2 und 5 Jahren sollte die Screen Time weniger als 1 Stunde pro Tag betragen, diese Stunde sollte auf gemeinsam wahrgenommene pädagogische hochwertige Angebote bezogen und begrenzt sein.

- Bei Kindern ab 6 Jahren sollten konsequente und gleichbleibende Begrenzungen der Mediennutzungszeiten erfolgen. Medien sollten generell nicht die zeitlichen Phasen für Schlaf, Sport oder andere gesundheitsbezogene Verhaltensweisen einnehmen.

- Keine Mediennutzung während der Mahlzeiten, medienfreie Räumlichkeiten (Schlafzimmer).

- Keine Mediennutzung, um Kinder zu beruhigen. Auch wenn die Mediennutzung als Überbrückung von Zeitabschnitten (Autofahrten, Flüge, medizinische Eingriffe) beruhigend wirken kann, besteht die Sorge, dass Kinder ihre eigenen Fähigkeiten, sich selbst zu beruhigen, nicht entwickeln.

- Nicht benutzte Medien sollten ausgeschaltet werden - kein im Hintergrund laufender Fernseher.

- Der Medienkonsum der Kinder sollte supervidiert werden, Apps und Programme sollten vor der Benutzung durch die Kinder selbst gesichtet bzw. angeschaut werden. 


\section{Empfehlungen an Erzieher in Kindergärten und Krippen}

Repräsentative Studien zeigen im Bereich der Medienverfügbarkeit eine zunehmende Digitalisierung in Kindertagesstätten auf. Unklar ist, in welchem zeitlichen Umfang, durch wen und wozu diese Medien verwendet werden: zum Erreichen wertvoller Bildungsziele oder zur unkreativen und einseitigen Beschäftigung der Kinder? Gleichermaßen unklar ist die medienpädagogische Kompetenz (vs. Orientierungslosigkeit und Überforderung) von Fachkräften in den Kindergärten oder -krippen.

Merke

Zurzeit dürften viele Leiter noch der Generation der Digital Immigrants angehören, während viele junge Fachkräfte bereits aus der Generation der Digital Natives stammen, was eine besondere Situation für die Implementierung digitaler Medien im Kindergarten darstellt.

Ob die Kindergartenkinder einen reflektierten, kritischen, selbstbestimmten und sinnvollen Umgang mit modernen elektronischen Medien erlernen können, bleibt offen und ist noch zu untersuchen. Ob im Kindergarten der Einsatz von Tablets, Computer und Internet überhaupt notwendig, sinnvoll und hilfreich ist, bleibt fraglich und ist ebenfalls zu untersuchen. Weitgehender Konsens besteht, dass im Krippenbereich (im Alter bis 2 Jahre) die Verwendung dieser modernen Medien nicht sinnvoll ist. Verbindliche Qualifizierungsrichtlinien, Bildungsziele, Verhaltensregeln und Nutzungsvereinbarungen fehlen weitgehend. Die Verantwortung von Fachkräften für die Medienmündigkeit in Kindergärten und Krippen ist zu reflektieren und zu fördern.

\section{Ausblick}

Der Medienmarkt unternimmt umfassende Anstrengungen in Bezug auf die jüngsten Zielgruppen, werden doch bei diesen hohes Wachstumspotenzial, eine frühe Bindung an digitale Medien und Gewinne vermutet. Gefragt ist also die konsequente und fachkundige Aufklärung von Eltern durch medizinische, psychologische, psychotherapeutische und pädagogische Fachkräfte über die gesundheitlichen Folgen eines zu frühen und exzessiven Medienkonsums. Darüber hinaus sind hier präventive Maßnahmen und Rahmensetzungen aus der Politik zu fordern, wie sie z. B. in der Raucherprävention erfolgreich eingesetzt worden sind.

Letztendlich ist zum aktuellen Zeitpunkt angesichts spärlich oder nicht vorhandener Studien für die frühe Kindheit zu empfehlen, in dieser vulnerablen Entwicklungsphase zurückhaltend bis restriktiv mit digitalen Spiel- und Kommunikationsgeräten umzugehen. Risiken im Säuglingsund Kleinkindalter lassen sich reduzieren, indem der Medienkonsum durch Eltern und Erzieher geringgehalten wird und vielmehr später ab dem letzten Kindergartenjahr (in der Schulvorbereitung) ermöglicht wird.

Ein reflektierter, kritischer, selbstbestimmter und sinnvoller Umgang mit den elektronischen/digitalen Medien und den über sie zugänglichen Inhalten im Sinne von Medienmündigkeit (und nicht allein einer an der Machbarkeit orientierten Medienkompetenz) ist das wünschenswerte und vorrangige Entwicklungsziel. Schließlich hat mit der digitalen Revolution die „Zweite Moderne“ für die Menschheit gerade erst begonnen und die Zielsetzung der Aufklärung nicht zum Rückzug gebracht.

\section{KERNAUSSAGEN}

- Die Mediennutzung spielt bereits in der frühen Kindheit eine große Rolle. So belegen repräsentative Studien hohe Nutzungszeiten im Vorschulalter. Besonders in den ersten 2 Lebensjahren ist aufgrund der Gehirnentwicklung und der zentralen Bedeutung der emotionalen Beziehung zu den Eltern von einer besonders vulnerablen Phase auszugehen.

- Neben der Reflexion des elterlichen Mediengebrauchs stehen bei einer effektiven Beratung zur Mediennutzung in der frühen Kindheit Überlegungen zur Entwicklungsförderung und die gemeinsame Erarbeitung von Entwicklungszielen im Mittelpunkt.

- Die Interaktion stellt einen der wichtigsten und am stärksten abgesicherten Faktoren für die Kindesentwicklung dar. Emotionale Bindung und emotionale Verfügbarkeit von Vertrauenspersonen sind dabei zentral.

- Ein Wissenstransfer durch elektronische Medien ist für die ersten 18 Lebensmonate nicht belegt.

- Studien belegen für das 1. Lebensjahr die Unterlegenheit von Medienangeboten für den Spracherwerb verglichen mit nicht digitalen Spielgruppenangeboten.

- In Kindergärten und Krippen ist eine zunehmende Digitalisierung feststellbar. Unklar ist, in welchem zeitlichen Umfang, durch wen und wozu diese Medien verwendet werden; diesbezügliche Standards und Studien fehlen.

- Entwicklungsziele sollten z. B. für einen Schulerfolg präzisiert werden. Von besonderer Relevanz sind Fähigkeiten wie Task Persistence, Impulskontrolle, emotionale Regulation, soziale Kompetenz und kreatives und flexibles Denken.

- Der routinierte Umgang mit Medien per se stellt für den Schulerfolg keine Voraussetzung dar.

- Fernsehkonsum im Alter von 6-18 Monaten korreliert mit dem Auftreten von Aggressivität und externalisierenden Verhaltensstörungen.

- Belegt ist in vielen Einzelstudien und in Metaanalysen ein Zusammenhang zwischen „Gaming Disorder“, depressiven sowie Angststörungen und ADHS/HKS. 
Interessenkonflikt

Die Autoren geben an, dass keine Interessenkonflikte vorliegen.

\section{Autorinnen/Autoren}

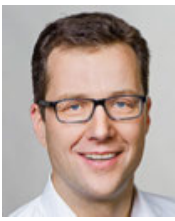

\section{Volker Mal}

Prof. Dr. med., seit 2012 ärztlicher Direktor des kbo-Kinderzentrums München und Lehrstuhlinhaber für Sozialpädiatrie an der Technischen Universität München. Vorstandsmitglied der Deutschen Gesellschaft für Sozialpädiatrie und Jugendmedizin e. V. Beiratstätigkeiten u. a. im Nationalen Zentrum Frühe Hilfen und im Deutschen Jugendinstitut. Aktuelle Forschungsschwerpunkte sind: Therapie der Zerebralparese, Früherkennung und -diagnostik im Rahmen der pädiatrischen Vorsorgeuntersuchungen, Entwicklung und Entwicklungsstörungen, psychosoziale Aspekte chronischer Erkrankungen.

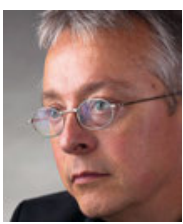

\section{Frank W. Paulus}

Dr. phil., seit 2009 Leitender Psychologe der Klinik für Kinder- und Jugendpsychiatrie, Psychosomatik und Psychotherapie am Universitätsklinikum des Saarlandes (UKS) Homburg/ Saar. Zuvor langjährige Berufserfahrungen in der Entwicklungspsychologie, Sozialpädiatrie, Erwachsenenpsychiatrie und Arbeitsmedizin. Psychologischer Psychotherapeut (VT), Kinder- und Jugendlichenpsychotherapeut (VT), Supervisor (VT) und Systemischer Therapeut (SGST). Therapie- und Forschungsschwerpunkte: Computerspiel- und Internetabhängigkeit, ADHS, Psychische Störungen im Vorschulalter.

\section{Korrespondenzadresse}

Prof. Dr. med. Volker Mall

Ärztlicher Direktor

kbo-Kinderzentrum München

Lehrstuhlinhaber Sozialpädiatrie, TU München

Heiglhofstr. 65

81377 München

chefsekretariat-kiz@kbo.de

\section{Wissenschaftlich verantwortlich} gemäß Zertifizierungsbestimmungen

Wissenschaftlich verantwortlich gemäß Zertifizierungsbestimmungen für diesen Beitrag ist Prof. Dr. med. Volker Mall, München.

\section{Literatur}

[1] Schmidt ME, Rich M, Rifas-Shiman SL et al. Television viewing in infancy and child cognition at 3 years of age in a US cohort. Pediatrics 2009; 123: 3

[2] Zimmerman FJ, Christakis DA, Meltzoff AN. Television and $\mathrm{DVD} /$ video viewing in children younger than 2 years. Arch Pediatr Adolesc Med 2007; 161: 473-479. doi:10.1001/archpedi.161.5.473
[3] Papousek M. Regulationsstörungen der frühen Kindheit: Klinische Evidenz für ein neues diagnostisches Konzept. In: Papousek M, Schieche M, Wurmser H, Hrsg. Regulationsstörungen der frühen Kindheit. Bern: Hans Huber 2004; 77-110

[4] Stern DN. The interpersonal World of the Infant. New York: Basic Books; 1985

[5] Campos JJ, Stenberg CR. Perception, Appraisal, and Emotions. The Onset of social Referencing. In: Lamb ME, Sherrod LR, eds. Infant social Cognition: empirical and social Considerations. Hillsdale: Erlbaum; 1981: 273-314

[6] Ainsworth MDS. Infancy in Uganda: Infant Care and the Growth of Love. Baltimore, MD: Johns Hopkins University Press; 1967

[7] Ainsworth MDS. Social Development in the first Year of Life: maternal Influences on Infant-mother Attachment. In: Tanner JM, ed. Developments in psychiatric Research: Essays based on the Sir Geoffrey Vickers Lectures of the Mental Health Foundation. London: Hodder \& Stoughton; 1977: 1-20

[8] Thalemann R, Wölfling K, Grüsser SM. Specific cue reactivity on computer-game related cues in excessive gamers. Behav Neurosci 2007; 121: 614-618

[9] Domingues-Montanari S. Clinical and psychological effects of excessive screen time on children. J Paed Child Health 2017; 53: $333-338$

[10] Takeuchi H, Taki Y, Hashizume H et al. The impact of television viewing on brain structures: Cross-sectional and longitudinal analyses. Cereb Cortex 2015; 25: 1188-1197. doi:10.1093/ cercor/bht315

[11] Li X, Atkins MS. Early childhood computer experience and cognitive and motor development. Pediatrics 2004; 113: 17151722. doi:10.1542/peds.113.6.1715

[12] Clawson A. The Bender Visual Motor Gestalt Test for Children. Los Angeles: Western Psychological Services; 1999

[13] Edelson LR, Mathias KC, Fulgoni VL 3rd et al. Screen-based sedentary behavior and associations with functional strength in 6-15 year old children in the United States. BMC Public Health 2016; 16: 116. doi:10.1186/s12889-016-2791-9

[14] Bedford R, Saez de Urabain IR, Cheung CHM et al. Toddlers' Fine Motor Milestone Achievement Is Associated with Early Touchscreen Scrolling. Front Psychol 2016; 7: 1108. doi:10.3389/fpsyg.2016.01108

[15] Maras D, Flamant MF, Murray M et al. Screen time is associated with depression and anxiety in Canadian youth. Prev Med. 2015; 73: 133-138. doi:10.1016/j.ypmed.2015.01.029

[16] Kremer P, Elshaug C, Leslie E et al. Physical activity, leisuretime screen use and depression among children and young adolescents. J Sci Med Sport 2014; 17: 183-187. doi:10.1016/j.jsams.2013.03.012

[17] Domingues-Montanari S. Clinical and psychological effects of excessive screen time on children. J Paediatr Child Health 2017; 53: 333-338. doi:10.1111/jpc.13462

[18] Radesky J, Christakis D, Hill D et al. Media and Young Minds. Pediatrics 2016; 138: e20162591

[19] Marinelli M, Sunyer J, Alvarez-Pedrerol M et al. Hours of television viewing and sleep duration in children: A multicenter birth cohort study. JAMA Pediatr 2014; 168: 458-464. doi:10.1001/jamapediatrics.2013.3861

[20] Zhang G, Wu L, Zhou L et al. Television watching and risk of childhood obesity: A meta-analysis. Eur J Public Health 2016; 26: 13-18. doi:10.1093/eurpub/ckv213

[21] Medienpädagogischer Forschungsverbund Südwest. miniKIM. Kleinkinder und Medien. Basisuntersuchung zum Medienum- 
gang 2- bis 5-Jähriger in Deutschland. Stuttgart: Forschungsberichte mpfs; 2014

[22] Medienpädagogischer Forschungsverbund Südwest. miniKIM. Kleinkinder und Medien. Basisuntersuchung zum Medienumgang 2- bis 5-Jähriger in Deutschland. Stuttgart: Forschungsberichte mpfs; 2012

[23] Ehmig SC, Reuter T. Vorlesen im Kinderalltag. Bedeutung des Vorlesens für die Entwicklung von Kindern und Jugendlichen und Vorlesepraxis in den Familien. Stiftung Lesen, gefördert vom BMBF; 2013

[24] World Health Organization. International Classification of Diseases 11th Revision ICD-11 Beta Draft 2017. Im Internet: https://icd.who.int/dev11/l-m/en; Stand: 30.04.2018

[25] Paulus FW. Computerspiel- und Internetabhängigkeit. Seelische Entwicklung in der virtuellen und realen Welt. Pädiat prax 2014; 82: 539-548

[26] Funk JB, Baldacci HB, Pasold T et al. Violence exposure in reallife, video games, television, movies, and the Internet: is there desensitization? J Adolesc 2004; 27: 23-39. doi:10.1016/j. adolescence.2003.10.005

[27] Uhlmann E, Swanson ]. Exposure to violent video games increases automatic aggressiveness. J Adolesc 2004; 27: 41-52. doi:10.1016/j.adolescence.2003.10.004

[28] Anderson CA, Bushman BJ. Effects of violent video games on aggressive behavior, aggressive cognition, aggressive affect, physiological arousal, and prosocial behavior: A meta-analytic review of the scientific literature. Psychol Sci 2001; 12: 353359. doi:10.1111/1467-9280.00366

[29] Anderson CA. An update on the effects of playing violent video games. J Adolesc 2004; 27: 113-122. doi:10.1016/j.adolescence.2003.10.009

[30] Carnagey NL, Anderson CA. The effects of reward and punishment in violent video games on aggressive affect, cognition, and behavior. Psychol Sci 2005; 16: 882-889. doi:10.1111/ j.1467-9280.2005.01632.x

[31] Gentile DA, Lynch PJ, Linder JR et al. The effects of violent video game habits on adolescent hostility, aggressive behaviors, and school performance. J Adolesc 2004; 27: 5-27. doi:10.1016/j.adolescence.2003.10.002

[32] Weaver E, Gradisar M, Dohnt $\mathrm{H}$ et al. The effect of presleep video-game playing on adolescent sleep. J Clin Sleep Med 2010; 6: 184-189

[33] Strube TB, In-Albon T, Weeß HG. Machen Smartphones Jugendliche und junge Erwachsene schlaflos? Somnologie 2016; 20: 61-66. doi:10.1007/s11818-016-0044-x

[34] Mendoza JA, Zimmerman FJ, Christakis DA. Television viewing, computer use, obesity, and adiposity in US preschool children. Int J Behav Nutr Phys Act 2007; 4: 44. doi:10.1186/14795868-4-44

[35] Ferguson C]. Do Angry Birds make for angry children? A metaanalysis of video game influences on childrens' and adolescents' aggression, mental health, prosocial behavior, and academic performance. Perspect Psychol Sci 2015; 10: 646-666. doi:10.1177/1745691615592234

[36] Ferguson C], Kilburn J. The public health risks of media violence: A meta-analytic review. J Pediat 2009; 154: 759-763. doi:10.1016/j.jpeds.2008.11.033

[37] Grüsser SM, Thalemann R, Griffiths MD. Excessive computer game playing: evidence for addiction and aggression? Cyberpsychol Behav 2007; 10: 290-292. doi:10.1089/ cpb.2006.9956

[38] Appel M, Schreiner C. Leben in einer digitalen Welt: Wissenschaftliche Befundlage und problematische Fehlschlüsse. Psy- chol Rundsch 2015; 66: 119-123. doi:10.1026/0033-3042| a000252

[39] Yau YHC, Potenza MN. Gambling Disorder and Other Behavioral Addictions: Recognition and Treatment. Harv Rev Psychiatry 2015; 23: 134-146. doi:10.1097/HRP.0000000000000051

[40] Fauth-Bühler M, Mann K. Neurobiological correlates of internet gaming disorder: Similarities to pathological gambling. Addict Behav 2015; 64: 349-356. doi:10.1016/j.addbeh.2015.11.004

[41] Chen CY, Yen JY, Wang PW et al. Altered Functional Connectivity of the Insula and Nucleus Accumbens in Internet Gaming Disorder: A Resting State fMRI Study. Eur Addict Res 2016; 22: 192-200. doi:10.1159/000440716

[42] Fineberg NA, Chamberlain SR, Goudriaan AE et al. New developments in human neurocognition: clinical, genetic, and brain imaging correlates of impulsivity and compulsivity. CNS Spectr 2014; 19: 69-89. doi:10.1017/S1092852913000801

[43] Meng $\mathrm{Y}$, Deng W, Wang $\mathrm{H}$ et al. The prefrontal dysfunction in individuals with Internet gaming disorder: a meta-analysis of functional magnetic resonance imaging studies. Addict Biol 2014; 20 :799-808. doi:10.1111/adb.12154

[44] Han DH, Bolo N, Daniels MA et al. Brain activity and desire for Internet video game play. Compr Psychiatry 2011; 52: 88-95. doi:10.1016/j.comppsych.2010.04.004

[45] Ko CH, Liuc GC, Yena JY et al. The brain activations for both cue-induced gaming urge and smoking craving among subjects comorbid with Internet gaming addiction and nicotine dependence. J Psychiatr Res 2013; 47: 486-493. doi:10.1016/j.jpsychires.2012.11.008

[46] Ko CH, Yen JY, Liua SC et al. The Associations between aggressive Behaviors and Internet Addiction and Online Activities in Adolescents. J Adolesc Health 2009; 44: 598-605. doi:10.1016/j.jadohealth.2008.11.011

[47] Leeman RF, Potenza MN. A Targeted Review of the Neurobiology and Genetics of Behavioral Addictions: An Emerging Area of Research. Can J Psychiatry 2013; 58: 260-273. doi:10.1177| 070674371305800503

[48] Weinstein A, Lejoyeux M. New Developments on the Neurobiological and Pharmaco-Genetic Mechanisms Underlying Internet and Videogame Addiction. Am J Addict 2015; 24: 117125. doi: $10.1111 /$ ajad. 12110

[49] Koob GF, Volkow ND. Neurocircuitry of Addiction. Neuropsychopharm 2010; 35: 217-238. doi:10.1038/npp.2009.110

[50] Atkin AJ, Sharp SJ, Corder K et al. International Children's Accelerometry Database (ICAD) Collaborators. Prevalence and correlates of screen time in youth: An international perspective. Am J Prev Med 2014; 47: 803-807

[51] Lillard AS, Drell MB, Richey EM et al. Further examination of the immediate impact of television on children's executive function. Dev Psychol 2015; 51: 792-805. doi:10.1037| a0039097

[52] Green CS, Bavelier D. Effect of action video games on the spatial distribution of visuospatial attention. J Exp Psychol Hum Percept Perform 2006; 32: 1465-1478. doi:10.1037/00961523.32.6.1465

[53] Subrahmanyam K, Greenfield P, Kraut R et al. The impact of computer use on childrens' and adolescents' development. J Appl Dev Psychol 2001; 22: 7-30. doi:10.1016/S0193-3973 (00)00063-0

[54] Lieberman DA. Management of chronic pediatric diseases with interactive health games: Theory and research findings. J Ambul Care Manage 2001; 24: 26-38 
[55] Klingberg T, Fernell E, Olesen P et al. Computerized training of working memory in children with ADHD - a randomized, controlled trial. J Am Acad Child Adolesc Psychiatry 2005; 44: 177-186

[56] Prins PJ, Dovis S, Ponsioen A et al. Does computerized working memory training with game elements enhance motivation and training efficacy in children with ADHD? Cyberpsychol Behav Soc Netw 2011; 14: 115-122. doi:10.1089/cyber.2009.0206

[57] Redd WH, Jacobsen PB, Die-Trill M et al. Cognitive-attentional distraction in the control of conditioned nausea in pediatric cancer patients receiving chemotherapy. J Consult Clin Psychol 1987; 55: 391-395. doi:10.1037/0022-006X.55.3.391

[58] Paulus FW. Kinderwelten im Wandel: Von der analogen zur digitalen Welt. Kinderärztl Prax 2018; 89: 121-128

[59] Paulus FW. Computerspielabhängigkeit und ADHS. In: Rösler M, Retz W, von Gontard A, Paulus FW, Hrsg. Soziale Folgen der ADHS. Stuttgart: Kohlhammer; 2014: 99-111

[60] Carli V, Durkee T, Wassermann D et al. The Association between pathological Internet Use and Comorbid Psychopathology: A Systematic Review. Psychopathology 2013; 46: 1-13

[61] Paulus FW, Sinzig J, Mayer $\mathrm{H}$ et al. Computer Gaming Disorder and ADHD in Young Children - a Population-Based Study. Int J Ment Health Add 2017; 1-15

[62] Vogelgesang S, Hessel S. Spionagegeräte im Kinderzimmer? Zur Problematik des §90 TKG bei Smart Toys. Z Datensch 2017; 6: 269-273
[63] Hessel S. „My friend Cayla“ - eine nach §90 TKG verbotene Sendeanlage? JurPC 2017; 13. doi:10.7328/jurpcb201732113

[64] Bleckmann P. Medienmündig - Wie unsere Kinder selbstbestimmt mit dem Bildschirm umgehen lernen. Stuttgart: Klett-Cotta; 2016

\section{Bibliografie}

DOI https://doi.org/10.1055/s-0043-115286

Pädiatrie up2date 2018; 13: 119-134

(c) Georg Thieme Verlag KG Stuttgart · New York ISSN 1611-6445 
Diese Fortbildungseinheit ist 12 Monate online für die Teilnahme verfügbar.

Sollten Sie Fragen zur Online-Teilnahme haben, finden Sie unter cme.thieme.de/hilfe eine ausführliche Anleitung. Wir wünschen viel Erfolg beim Beantworten der Fragen!

Unter eref.thieme.de/ZZX8XXM oder über den QR-Code kommen Sie direkt zum Artikel zur Eingabe der Antworten.

VNR 2760512018154654321

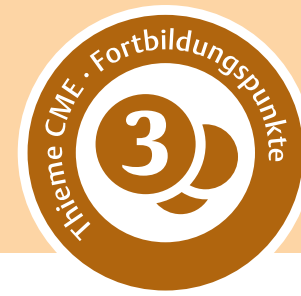

\section{Frage 1}

Welches ist kein bedeutsames Konzept der frühen Eltern-KindInteraktion?
A Feinfühligkeit
B Social Referencing
C elterliche Kompetenzen
D affektive Abstimmung
E Screen Time

\section{Frage 2}

Welche Aussage bezüglich der Entwicklung ist falsch?

A Der Kopf des Neugeborenen wächst beim Menschen um nur noch $30 \%$.

B Das Becken ist relativ gesehen beim Menschen aus statischen Gründen kleiner als bei Schimpansen.

C Die Interaktion stellt einen wichtigen Stimulus für die Ausbildung des frühkindlichen neuronalen Netzwerks dar.

D Konzepte der frühen Interaktion stellen die intuitiven elterlichen Kompetenzen, die affektive Abstimmung und die soziale Rückversicherung dar.

E Der Einfluss von sozialer Interaktion auf die Gehirnentwicklung ist beim Menschen besonders ausgeprägt.

\section{Frage 3}

Welche Aussage zur Feinfühligkeit ist nach Ainsworth (1967, 1977) falsch?

A Sie bezieht sich ausschließlich auf die Mutter-Kind-Interaktion.

B Sie beschreibt die Wahrnehmung der Signale des Säuglings durch eine Bezugsperson.

C Sie bezieht sich auch auf deren Interpretation und die Reaktion.

D Sie kann durch psychische Erkrankungen beeinträchtigt sein.

E Sie bedingt eine kurze Reaktionslatenz insbesondere auf Quengeln und Weinen des Säuglings.

\section{Frage 4}

Welche Aussage ist richtig?

A Ab dem 6. Lebensmonat reagieren Säuglinge differenziert auf die Muttersprache.

B Akustische und visuelle Stimuli von Computerspielen zeigen im EEG bei Erwachsenen ähnliche Muster wie suchtmittelspezifische Reize.

C Das Prinzip der klassischen Konditionierung greift bei der exzessiven Nutzung von Computerspielen nicht.

D Neurobiologische Anomalien sind im Zusammenhang mit exzessivem Computerspielen nicht zu finden.

E Exzessiver Medienkonsum während der Schwangerschaft ist nachgewiesenermaßen für den Fötus schädlich.

\section{Frage 5}

Welche Aussage ist richtig?

A Pädagogisch hochwertige Angebote können bereits im 1. Lebensjahr zum Spracherwerb beitragen.

B Ende des 2. Lebensjahres ist eine Gleichwertigkeit der Wissensvermittlung von pädagogisch hochwertigen elektronischen Medien mit konventionellen analogen Angeboten belegt.

C In mehrsprachigen Spielgruppen kann im 1. Lebensjahr bereits nach wenigen Stunden eine veränderte (der Muttersprache ähnelnde) Reaktion auf fremdsprachliche Laute festgestellt werden.

D Mediennutzung zur kurzfristigen Zeitüberbrückung ohne Begleitung einer Bezugsperson ist auch in den ersten 2 Lebensjahren unbedenklich.

E Das Spielen von Kleinkindern unter Einbeziehung einer erwachsenen Bezugsperson mit elektronischen Medien ist nach aktueller Studienlage wahrscheinlich dem Spielen mit analogen Spielen (unter Einbeziehung einer erwachsenen Bezugsperson) gleichzusetzen.

\section{- Weitere Fragen auf der folgenden Seite...}


Punkte sammeln auf CMI. thilemede

Fortsetzung...

\section{Frage 6}

Welche Aussage ist falsch? In mehreren Studien und einer Metaanalyse konnte gezeigt werden, dass ab 1 Stunde Fernsehen pro Tag ...

A das Schlafverhalten beeinträchtigt ist.

B sich das Adipositasrisiko erhöht.

C der Nahrungsanteil von Gemüse und Obst sinkt.

D der Konsum von zucker- und fetthaltigen Nahrungsmitteln steigt.

E negative Effekte vor allem auf den ökonomischen Status und das Bildungsniveau zurückzuführen sind.

\section{Frage 7}

Welche folgende Empfehlung einer pädiatrischen Fachgesellschaft zum Medienkonsum liegt tatsächlich vor?

A USA: „Null Konsum“ in den ersten 18 Lebensmonaten

B Europäische Union: „Null Konsum“ in den ersten 12 Monaten

C Deutsche Akademie für Kinderheilkunde und Jugendmedizin: „Null Konsum“ in den ersten 2 Lebensjahren

D USA: ab dem 2. Lebensjahr unter 1 Stunde

E Australien: ab dem 2. Lebensjahr unter 2 Stunden

\section{Frage 8}

Welche Aussage zu Computerspiel- und Internetabhängigkeit ist falsch?

A Sie lässt sich über die ICD-10 nicht kodieren.

B Sie lässt sich über die DSM-5 kodieren.

C Sie wird in der ICD-11 voraussichtlich nicht berücksichtigt.

D Sie geht mit Entzugssymptomen wie Reizbarkeit, Ängstlichkeit, Traurigkeit einher.

E Sie führt zum Interessensverlust an früheren Hobbys und Freizeitbeschäftigungen.

\section{Frage 9}

Was sollte eine Elternberatung zum Thema Mediennutzung beinhalten?

A Eine Aufklärung über die Phasen der Synaptogenese.

B Die Relevanz von Interaktion für die kindliche Entwicklung.

C Den Hinweis, dass frühe Mediennutzung für die Gehirnentwicklung nützlich sein kann.

D Positive Erfahrungen über die Mediennutzung im Jugendund Erwachsenenalter sind auch in das Kleinkindalter übertragbar.

E Frühzeitige Gewöhnung an elektronische Medien stellt eine präventive Maßnahme gegenüber Medien- oder Internetsucht da.

\section{Frage 10}

Welche Aussage zum Fernsehkonsum ist falsch?

A Fernsehkonsum im Alter von 6-18 Monaten geht mit einer erhöhten Wahrscheinlichkeit für externalisierte Verhaltensstörungen einher.

B Fernsehkonsum korreliert negativ mit den Schulleistungen.

C Die sog. „Screen Time“ korreliert mit dem Auftreten von Angststörungen und Depressionen.

D Das Einhalten einer Konsumobergrenze von 1 Stunde Fernsehen pro Tag reduziert die Wahrscheinlichkeit für das Auftreten einer Depression.

E Ein geringer Fernsehkonsum erhöht das Risiko für Mobbing durch Gleichaltrige. 